J. Korean Math. Soc. 51 (2014), No. 1, pp. 137-162

http://dx.doi.org/10.4134/JKMS.2014.51.1.137

\title{
CONVERGENCE OF THE RELAXED NEWTON'S METHOD
}

\author{
Ioannis Konstantinos Argyros, José Manuel Gutiérrez, \\ Ángel Alberto Magreñán, and Natalia Romero
}

\begin{abstract}
In this work we study the local and semilocal convergence of the relaxed Newton's method, that is Newton's method with a relaxation parameter $0<\lambda<2$. We give a Kantorovich-like theorem that can be applied for operators defined between two Banach spaces. In fact, we obtain the recurrent sequence that majorizes the one given by the method and we characterize its convergence by a result that involves the relaxation parameter $\lambda$. We use a new technique that allows us on the one hand to generalize and on the other hand to extend the applicability of the result given initially by Kantorovich for $\lambda=1$.
\end{abstract}

\section{Introduction}

In many areas related to the applied sciences one confronts the problem of solving a nonlinear equation of the form $f(x)=0$. The solutions of these equations can rarely be found in closed form. That is why most solution methods are iterative. There exist lots of iterative methods with different properties that allow us to solve this kind of equations, but the most well-known and used is the Newton's method, which has the following form:

$$
x_{n+1}=x_{n}-\frac{f\left(x_{n}\right)}{f^{\prime}\left(x_{n}\right)}, \quad n \geq 0 .
$$

In the beginning, this method was constructed for functions defined on the real line, but after the seminal works of Kantorovich [16], Newton's method was extended to general spaces with the aim of solving functional equations in the form

$$
F(x)=0,
$$

where $F: X \rightarrow Y$ is a function defined between two Banach spaces (of [18], [27], or [28] for more details). In this way, the method could be used to solve different kind of problems such as systems of nonlinear equations, nonlinear

Received March 21, 2013.

2010 Mathematics Subject Classification. 65J15, 47H17, 65H10, 65G99, 49M15.

Key words and phrases. relaxed Newton's method, Banach space, Kantorovich hypothesis, majorizing sequence, local convergence, semilocal convergence. 
integral equations, ordinary and partial differential equations or variational problems.

In this context, the known as Newton-Kantorovich method has the following form:

$$
x_{n+1}=x_{n}-\Gamma_{n} F\left(x_{n}\right), \quad n \geq 0,
$$

where $\Gamma_{n}=F^{\prime}\left(x_{n}\right)^{-1}$ is the inverse of the Fréchet derivative of the nonlinear operator $F(x)$ at the point $x_{n}$.

The study about convergence matter of iterative methods is usually based on two types: semilocal and local convergence analysis. The semilocal matter is, based on the information around an initial point, to give conditions ensuring the convergence of the iterative method, while the local one is, based on the information around a solution, to find estimates of the radii of convergence balls.

In order to ensure that the method is well defined and that converges to a solution of the equation (2), Kantorovich and other authors (see [16], [19], $[20])$ give sufficient conditions on the starting point $x_{0}$ and on the operator $F$. The main convergence result for this method was given by Kantorovich in [16]. Here and in the rest of the paper, we denote

$$
B\left(x_{0}, R\right)=\left\{x:\left\|x-x_{0}\right\|<R\right\} .
$$

Theorem 1 (Kantorovich's theorem). Let us assume that the operator $F$ introduced in (2) is defined and Fréchet differentiable in a ball $B\left(x_{0}, R\right)$. Let us assume that the linear operator $F^{\prime}\left(x_{0}\right)$ is invertible and conditions

$$
\begin{gathered}
\left\|F^{\prime}\left(x_{0}\right)^{-1} F\left(x_{0}\right)\right\| \leq a, \\
\left\|F^{\prime}\left(x_{0}\right)^{-1}\left[F^{\prime}(x)-F^{\prime}(y)\right]\right\| \leq b\|x-y\|, \quad x, y \in B\left(x_{0}, R\right),
\end{gathered}
$$

hold. In addition, let us suppose,

$$
h=a b<1 / 2, \quad u_{1}=\frac{1-\sqrt{1-2 h}}{b} \leq R .
$$

Then, Newton-Kantorovich method (3) is well defined and it converges to a solution $x^{*}$ of $(2)$. In addition, $x^{*}$ is located in $\overline{B\left(x_{0}, u_{1}\right)}$ an it is the unique solution of $(2)$ in the ball $B\left(x_{0}, u_{2}\right)$, where

$$
u_{2}=\frac{1+\sqrt{1-2 h}}{b} .
$$

Finally, the rate of convergence is given by

$$
\left\|x^{*}-x_{n}\right\| \leq \frac{a}{h 2^{n}}(2 h)^{2^{n}}, \quad n \geq 0 .
$$

That is, the order of convergence is quadratic.

We would like to emphasize the key role of the parameter $h$ defined above in Kantorovich's theory. The previous result has been stated for values $h<1 / 2$, but Kantorovich theory can be applied for $h \leq 1 / 2$. When $h=1 / 2$, only the 
linear convergence can be guaranteed. In addition, in this case, $u_{1}=u_{2}$, and the solution $x^{*}$ is located and is unique in $\overline{B\left(x_{0}, u_{1}\right)}$.

Our main goal in this paper is to develop a Kantorovich-like theory for a variant of Newton's method that it is called relaxed Newton's method:

$$
x_{n+1}=x_{n}-\lambda F^{\prime}\left(x_{n}\right)^{-1} F\left(x_{n}\right), \quad n \geq 0, \quad 0<\lambda<2 .
$$

The main reason to consider this method is because a full Newton step may not be suitable to ensure that convergence of the method is monotone, but introducing the relaxing factor this problem disappears. Some questions about the complex dynamics of this method have been considered in [14] or [17]. Note also that method (6) is a special case of Inexact Newton's method defined by:

$$
x_{n+1}=x_{n}-F^{\prime}\left(x_{n}\right)^{-1} F\left(x_{n}\right)+y_{n},
$$

where $y_{n}$ is a null residual sequence in $X$. In particular, if

$$
y_{n}=(1-\lambda) F^{\prime}\left(x_{n}\right)^{-1} F\left(x_{n}\right)
$$

the Inexact Newton's method reduces to method (6). There is a plethora of convergence results for the Inexact Newton's method (cf [2]-[6], [8]-[15], [17], [18], [23], [24], [26], [29] and the references there in). Hence, the results for Inexact Newton's method can be specialized to provide convergence results for method (6). However, we decided in this paper to employ a more direct approach which leads to easier to verify sufficient convergence conditions under simpler hypotheses.

In [8] we can find a semilocal convergence analysis of methods in the form (6) but it is related to recurrent sequences and functional spaces.

The relevance of our study, compared with the classical Kantorovich theory, is the inclusion of the relaxation parameter $\lambda$ in the set of sufficient conditions to guarantee the semilocal convergence of (6) to a solution of (2). To be more precise, throughout this paper we assume that the operator $F$ defined in (2) is defined and Fréchet differentiable in a ball $B\left(x_{0}, R\right)$.

The paper is organized as follows. In Section 2 we study the local convergence of the method under Lipschitz and center-Lipschitz conditions. In Section 3 the semilocal convergence of the method is studied under only Lipschitz conditions and $\lambda \in(0,1)$. The semilocal convergence of the method under center-Lipschitz and Lipschitz conditions for $\lambda \in(0,2)$ is given in Section 4 . We present an extended semilocal convergence analysis for method (6) in Section 5 containing a nondifferentiable term. Finally, Section 6 is devoted to some examples and numerical experiments to illustrate the theoretical results given in the previous sections.

\section{Local convergence of the relaxed Newton's method}

In this section we will study the local convergence of the relaxed Newton's method under center-Lipschitz and Lipschitz conditions. We present the following result. 
Theorem 2. Let $F: X \rightarrow Y$ be Fréchet differentiable in the ball $B\left(x^{*}, R\right) \subseteq X$. Assume that the following conditions hold:

(i) There exists a solution $x^{*}$ of $F(x)=0$.

(ii) There exists $\Gamma=F^{\prime}\left(x^{*}\right)^{-1}$.

(iii) $\left\|\Gamma\left[F^{\prime}(x)-F^{\prime}(y)\right]\right\| \leq b\|x-y\|, b>0, x, y \in B\left(x^{*}, R\right)$.

(iv) $\left\|\Gamma\left[F^{\prime}(x)-F^{\prime}\left(x^{*}\right)\right]\right\| \leq \beta\left\|x-x^{*}\right\|, \beta>0, x \in B\left(x^{*}, R\right)$.

(v) $\rho=\min \left\{R, \frac{2(1-|1-\lambda|)}{b+(2+|1-\lambda|) \beta}\right\}, 0<\lambda<2$.

Then, the sequence given by the relaxed Newton's method (6) starting from $x_{0} \in B\left(x^{*}, \rho\right)$, remains in $B\left(x^{*}, \rho\right)$ and converges to $x^{*}$.

Proof. We need to prove the following conditions:

(I) If $x \in B\left(x^{*}, \rho\right)$, then

$$
\beta\left\|x-x^{*}\right\| \leq \beta \rho<1,
$$

by the choice of $\rho$ given in $(\mathrm{v})$. Hence,

$$
\left\|I-\Gamma F^{\prime}(x)\right\| \leq \beta\left\|x-x^{*}\right\|<1 .
$$

By the Banach Lemma on inversion of operators ([7], [16], [19]-[22]) we can ensure that there exists the inverse operator of $\Gamma F^{\prime}(x)$ and

$$
\left\|F^{\prime}(x)^{-1} F^{\prime}\left(x^{*}\right)\right\| \leq \frac{1}{1-\beta\left\|x-x^{*}\right\|} .
$$

(II) We have the Ostrowski decomposition:

$$
\begin{aligned}
F(x) & =F(x)-F\left(x^{*}\right)-F^{\prime}\left(x^{*}\right)\left(x-x^{*}\right)+F^{\prime}\left(x^{*}\right)\left(x-x^{*}\right) \\
& =\int_{x^{*}}^{x}\left[F^{\prime}(x)-F^{\prime}\left(x^{*}\right)\right] d x+F^{\prime}\left(x^{*}\right)\left(x^{*}-x\right) \\
& =\int_{0}^{1}\left[F^{\prime}\left(x^{*}+t\left(x-x^{*}\right)\right)-F^{\prime}\left(x^{*}\right)\right]\left(x-x^{*}\right) d t+F^{\prime}\left(x^{*}\right)\left(x^{*}-x\right) .
\end{aligned}
$$

By taking norms in the preceding identity and using (iv) we get that

$$
\|\Gamma F(x)\| \leq \frac{\beta}{2}\left\|x-x^{*}\right\|^{2}+\left\|x-x^{*}\right\| .
$$

We also have the following Ostrowski-type decomposition:

$$
\begin{aligned}
& x^{*}-x_{n+1} \\
= & x^{*}-x_{n}+\lambda \Gamma_{n} F\left(x_{n}\right)-\Gamma_{n} F\left(x_{n}\right)+\Gamma_{n} F\left(x_{n}\right) \\
= & x^{*}-x_{n}+\Gamma_{n} F\left(x_{n}\right)+(\lambda-1) \Gamma_{n} F\left(x_{n}\right) \\
= & -\Gamma_{n}\left[F\left(x^{*}\right)-F\left(x_{n}\right)-F^{\prime}\left(x_{n}\right)\left(x^{*}-x_{n}\right)+(1-\lambda) F\left(x_{n}\right)\right] \\
= & -\Gamma_{n}\left[\int_{x_{n}}^{x^{*}}\left[F^{\prime}(x)-F^{\prime}\left(x_{n}\right)\right] d x+(1-\lambda) F\left(x_{n}\right)\right] \\
= & -\Gamma_{n}\left[\int_{0}^{1}\left[F^{\prime}\left(x_{n}+t\left(x^{*}-x_{n}\right)\right)-F^{\prime}\left(x_{n}\right)\right]\left(x^{*}-x_{n}\right) d t+(1-\lambda) F\left(x_{n}\right)\right] .
\end{aligned}
$$


Then, by taking norms we get in turn that:

$$
\begin{aligned}
&\left\|x^{*}-x_{n+1}\right\| \\
& \leq\left\|\Gamma_{n} F^{\prime}\left(x^{*}\right)\right\|\left[\frac{b}{2}\left\|x^{*}-x_{n}\right\|^{2}+(1-\lambda)\left\|\Gamma F\left(x_{n}\right)\right\|\right] \\
& \leq \frac{1}{1-\beta\left\|x^{*}-x_{n}\right\|}\left[\frac{b}{2}\left\|x^{*}-x_{n}\right\|^{2}+(1-\lambda)\left(\frac{\beta}{2}\left\|x^{*}-x_{n}\right\|^{2}+\left\|x^{*}-x_{n}\right\|\right)\right] \\
& \leq \frac{1}{1-\beta\left\|x^{*}-x_{n}\right\|}\left[\left(\frac{b}{2}+\beta\left(\frac{1-\lambda}{2}\right)\right)\left\|x^{*}-x_{n}\right\|+(1-\lambda)\right]\left\|x^{*}-x_{n}\right\| . \\
& \text { Let } a_{n+1}=\frac{a_{n}}{1-\beta a_{n}}\left(\left(\frac{|1-\lambda|}{2} \beta+\frac{b}{2}\right) a_{n}+|1-\lambda|\right) . \text { Then, } \\
&\left\|x^{*}-x_{n}\right\| \leq a_{n} \forall n .
\end{aligned}
$$

Let $\gamma=\frac{\beta}{b}$ and $\epsilon_{n}=\beta a_{n}$ :

$$
\epsilon_{n+1}=\frac{\epsilon_{n}}{1-\epsilon_{n}}\left(\frac{\frac{1}{\gamma}+|1-\lambda|}{2} \epsilon_{n}+|1-\lambda|\right) .
$$

Using an induction process, we will prove that $\left(\epsilon_{n}\right)$ converges to 0 . Let's show that $\epsilon_{1}<\epsilon_{0}$ :

or

$$
\epsilon_{1}=\frac{\epsilon_{0}}{1-\epsilon_{0}}\left(\frac{\frac{1}{\gamma}+|1-\lambda|}{2} \epsilon_{0}+|1-\lambda|\right)<\epsilon_{0}
$$

$$
\epsilon_{0}<\frac{2(1-|1-\lambda|)}{2+\frac{1}{\gamma}+|1-\lambda|},
$$

which is true by the choice of $\rho$. Supposing that $\epsilon_{n}<\epsilon_{n-1} \forall n \leq k$. Let's see that it remains valid for $n=k+1$. It's easy to see that $\epsilon_{n}<\epsilon_{n-1}$ and $1-\epsilon_{n}>1-\epsilon_{n-1}$. Moreover as $0<\lambda<2, \frac{1}{\gamma}+|1-\lambda|>0$, so $\epsilon_{k+1}<\epsilon_{k}$. Clearly from the above proof and $x_{0} \in B\left(x^{*}, \rho\right)$ it follows that $x_{n} \in B\left(x^{*}, \rho\right) \forall n \geq 0$ The proof is complete.

Remark 1. Hypothesis (iv) is not additional to (iii), since (iii) always implies (iv). We also have that

$$
\beta \leq b
$$

and $\frac{b}{\beta}$ can be arbitrarily large. Indeed, let us consider as an example $X=Y=$ $\mathbb{R}, x^{*}=0$ and define the function $F$ on $X$ by

$$
F(x)=d_{0} x-d_{1} \sin (1)+d_{1} \sin \left(e^{d_{2} x}\right),
$$

where $d_{0}, d_{1}, d_{2}$ are given parameters. Then, it can easily be seen that for $d_{2}$ sufficiently large and $d_{1}$ sufficiently small, $b / \beta$ can be arbitrarily large. Other examples where $\beta<b$ can be found in [7]-[9]. 
If hypothesis (iv) is dropped, then the convergence radius can be defined by

$$
\rho_{0}=\min \left\{R, \frac{2(1-|1-\lambda|)}{(3+|1-\lambda|) b}\right\} .
$$

Notice that

$$
\rho_{0} \leq \rho .
$$

The preceding inequality can be strict if $\beta<b$ and

$$
\frac{2(1-|1-\lambda|)}{(3+|1-\lambda|) b}<R
$$

If $\lambda=1$ (Newton's method case), then $\rho, \rho_{0}$ reduce, respectively, to

$$
\rho^{A}=\min \left\{R, \frac{2}{2 \beta+b}\right\}
$$

and

$$
\rho_{0}^{T R}=\min \left\{R, \frac{2}{3 b}\right\}
$$

The radius $\rho^{A}$ was given by Argyros [6]-[9], whereas $\rho^{T R}$ was given independently by Rheinboldt [19] and Traub [25]. Clearly we have again that

$$
\rho^{T R} \leq \rho^{A}
$$

The preceding inequality is strict if $\beta<b$.

\section{Semilocal convergence under Lipschtiz conditions}

In this section our main purpose is to study the semilocal convergence of the relaxed Newton's method defined in (6) by means of using similar techniques as Kantorovich did for Newton's method. First of all, we present the following lemma.

Lemma 1. Let $p(r)$ be the polynomial:

$$
p(r)=\frac{b}{2} r^{2}-r+a
$$

with $a, b>0, a b<1 / 2, \lambda \in(0,1)$ and let be $r_{0}=0$. Then, the sequence given by $r_{n+1}=N_{\lambda, p}\left(r_{n}\right)$ converges to

$$
r^{*}=\frac{1-\sqrt{1-2 a b}}{b},
$$

where

$$
N_{\lambda, p}\left(r_{n}\right)=r_{n}-\lambda \frac{p\left(r_{n}\right)}{p^{\prime}\left(r_{n}\right)} .
$$


Proof. We begin by proving that $\left(r_{n}\right)$ is an increasing sequence:

$$
r_{1}=r_{0}-\lambda \frac{p\left(r_{0}\right)}{p^{\prime}\left(r_{0}\right)}=\lambda a>0=r_{0} .
$$

Let suppose that $r_{n}>r_{n-1}, \forall n \leq k-1$. We shall show that $r_{n+1}>r_{n}$. As $p(r)>0, p^{\prime}(r)<0, \forall r \in\left[0, r^{*}\right]$, we deduce that $r_{n+1}>r_{n}$. So $\left(r_{n}\right)$ is an increasing sequence. Secondly, using an induction process we see that $r^{*}$ is the upper bound of the sequence $\left(r_{n}\right)$. Indeed, it's immediate that:

$$
r^{*}-r_{0}=r^{*}>0 \text {. }
$$

Let's suppose that $r^{*}-r_{k}>0$. We shall prove that the preceding inequality is true for prove that $n=k+1$ :

$$
r^{*}-r_{k+1}=N_{\lambda, p}\left(r^{*}\right)-N_{\lambda, p}\left(r_{k}\right) .
$$

Taking into account the mean value theorem we have that:

$$
N_{\lambda, p}\left(r^{*}\right)-N_{\lambda, p}\left(r_{k}\right)=N_{\lambda, p}^{\prime}(\xi)\left(r^{*}-r_{k}\right), \xi \in\left(r_{k}, r^{*}\right) .
$$

As $r^{*}-r_{k}>0$, if $N_{\lambda, p}^{\prime}(\xi)>0$ the proof is ended, but:

$$
N_{\lambda, p}^{\prime}(\xi)=(1-\lambda)+\lambda \frac{p(\xi) p^{\prime \prime}(\xi)}{p^{\prime}(\xi)^{2}} .
$$

As $p(z)>0$ and $p^{\prime \prime}(z)>0, \forall \xi \in\left(0, r^{*}\right)$ and $\lambda \in(0,1)$ then $N_{\lambda, p}^{\prime}(z)>0$. Hence, $\left(r_{n}\right)$ is bounded and so $\left(r_{n}\right)$ converges to its unique least upper bound $r_{0}^{*}<r^{*}$. Taking limits in $(10)$, it's clear that $p\left(r_{0}^{*}\right)=0$, then $r_{0}^{*}=r^{*}$. The proof is complete.

Using this lemma and majorizing sequences we present the following result:

Theorem 3. Let $F: X \rightarrow Y$ be an operator defined between two Banach spaces $X, Y$, and Fréchet differentiable in the ball $B\left(x_{0}, R\right)$. Suppose that, the following conditions are satisfied:

(i) There exists the inverse operator of $F$ at the point $x_{0}$, moreover we will denote it by $\Gamma_{0}=F^{\prime}\left(x_{0}\right)^{-1}$,

(ii) $\left\|\Gamma_{0} F\left(x_{0}\right)\right\| \leq a, a>0$,

(iii) $\left\|\Gamma_{0}\left[F^{\prime}(x)-F^{\prime}(y)\right]\right\| \leq b\|x-y\|, b>0, x, y \in B\left(x_{0}, R\right)$,

(iv) $h=a b<1 / 2$, and

(v) $r^{*}=\frac{1-\sqrt{1-2 h}}{b} \leq R$.

Then, the sequence defined by:

$$
\left\{\begin{array}{l}
r_{0}=0 \\
r_{n+1}=r_{n}-\lambda \frac{p\left(r_{n}\right)}{p^{\prime}\left(r_{n}\right)}, n \geq 0,
\end{array}\right.
$$

where $p(r)$ is defined in (8), majorizes the sequence generated by the relaxed Newton's method. Moreover sequence $\left(x_{n}\right)$ converges to $x^{*}$ solution of $F(x)=0$. 
Limit point $x^{*}$ is located in $\overline{B\left(x_{0}, r^{*}\right)}$, where $r^{*}$ is defined in (9) and is unique in $B\left(x_{0}, r^{* *}\right)$, where

$$
r^{* *}=\frac{1+\sqrt{1-2 h}}{b} .
$$

Proof. First of all taking into account that $p^{\prime}(r)=b r-1$ we can define the sequence $\left(r_{n}\right)$ as:

$$
\left\{\begin{array}{l}
r_{0}=0 \\
r_{n+1}=r_{n}-\lambda \frac{\frac{b}{2} r_{n}^{2}-r_{n}+a}{b r_{n}-1}, n \geq 0 .
\end{array}\right.
$$

Lemma 1 ensures that the sequence $\left(r_{n}\right)$ is increasing and converges to $r^{*}$. We shall show that the following conditions are satisfied for every $n \in \mathbb{N}$ :

$\left(I_{n}\right)\left\|F^{\prime}\left(x_{n}\right)^{-1} F^{\prime}\left(x_{0}\right)\right\| \leq \frac{p^{\prime}\left(r_{0}\right)}{p^{\prime}\left(r_{n}\right)}$.

$\left(I I_{n}\right)\left\|\Gamma_{0} F\left(x_{n}\right)\right\| \leq-\frac{p\left(r_{n}\right)}{p^{\prime}\left(r_{0}\right)}$.

$\left(I I I_{n}\right)\left\|x_{n+1}-x_{n}\right\| \leq r_{n+1}-r_{n}$.

If $n=0$, it's easy to see that:

$\left(I_{0}\right)\left\|\Gamma_{0} \Gamma_{0}^{-1}\right\|=1=\frac{p^{\prime}\left(r_{0}\right)}{p^{\prime}\left(r_{0}\right)}$.

$\left(I I_{0}\right)\left\|\Gamma_{0} F\left(x_{0}\right)\right\| \leq a=-\frac{p\left(r_{0}\right)}{p^{\prime}\left(r_{0}\right)}$.

$\left(I I I_{0}\right)\left\|x_{1}-x_{0}\right\| \leq \lambda\left\|\Gamma_{0} F\left(x_{0}\right)\right\| \leq \lambda a=r_{1}-r_{0}$.

Suppose that items $\left(I_{n}\right)-\left(I I I_{n}\right)$ are true for $n \leq k-1$. We must show that these items are true for $n=k$. Taking into account that $\forall x \in B\left(x_{0}, r^{*}\right) \subseteq$ $B\left(x_{0}, R\right)$ it's clear that:

$$
\left\|I-\Gamma_{0} F^{\prime}(x)\right\| \leq\left\|\Gamma_{0}\left[F^{\prime}(x)-F^{\prime}\left(x_{0}\right)\right]\right\| \leq b\left\|x-x_{0}\right\|<b r^{*} \leq 1 .
$$

Consequently, by the Banach Lemma on inversion of operators:

We also have:

$$
\left\|F^{\prime}(x)^{-1} F^{\prime}\left(x_{0}\right)\right\| \leq \frac{1}{1-b\left\|x-x_{0}\right\|} .
$$

$$
\left\|x_{k}-x_{0}\right\| \leq\left\|x_{k}-x_{k-1}\right\|+\cdots+\left\|x_{1}-x_{0}\right\| \leq r_{k}-r_{0}=r_{k}<r^{*},
$$

so $x_{k} \in B\left(x_{0}, r^{*}\right)$. Now we consider the following Ostrowski-type decomposition:

$$
\text { (13) } \begin{aligned}
& F\left(x_{k+1}\right) \\
= & F\left(x_{k+1}\right)-\lambda F\left(x_{k}\right)-F^{\prime}\left(x_{k}\right)\left(x_{k+1}-x_{k}\right)-F\left(x_{k}\right)+F\left(x_{k}\right) \\
= & F\left(x_{k+1}\right)-F\left(x_{k}\right)-F^{\prime}\left(x_{k}\right)\left(x_{k+1}-x_{k}\right)+(1-\lambda) F\left(x_{k}\right) \\
= & \int_{x_{k}}^{x_{k+1}}\left[F^{\prime}(x)-F^{\prime}\left(x_{k}\right)\right] d x+(1-\lambda) F\left(x_{k}\right) \\
= & \int_{0}^{1}\left[F^{\prime}\left(x_{k}+t\left(x_{k+1}-x_{k}\right)\right)-F^{\prime}\left(x_{k}\right)\right]\left(x_{k+1}-x_{k}\right) d t+(1-\lambda) F\left(x_{k}\right)
\end{aligned}
$$


and so:

$$
\left\|\Gamma_{0} F\left(x_{k+1}\right)\right\| \leq \frac{b}{2}\left\|x_{k+1}-x_{k}\right\|^{2}+(1-\lambda)\left\|\Gamma_{0} F\left(x_{k}\right)\right\| .
$$

Moreover, repeating the process:

$$
\begin{aligned}
p\left(r_{k+1}\right) & =p\left(r_{k+1}\right)-\lambda p\left(r_{k}\right)-p^{\prime}\left(r_{k}\right)\left(r_{k+1}-r_{k}\right)-p\left(r_{k}\right)+p\left(r_{k}\right) \\
& =p\left(r_{k+1}\right)-p\left(r_{k}\right)-p^{\prime}\left(r_{k}\right)\left(r_{k+1}-r_{k}\right)+(1-\lambda) p\left(r_{k}\right) \\
& =\int_{r_{k}}^{r_{k+1}}\left[p^{\prime}(x)-p^{\prime}\left(r_{k}\right)\right] d x+(1-\lambda) p\left(r_{k}\right) \\
& =\int_{r_{k}}^{r_{k+1}} b\left(x-r_{k}\right) d x+(1-\lambda) p\left(r_{k}\right) \\
& =\frac{b}{2}\left(r_{k+1}-r_{k}\right)^{2}+(1-\lambda) p\left(r_{k}\right) .
\end{aligned}
$$

Furthermore, we have that

$\left(I_{k}\right)\left\|F^{\prime}\left(x_{k}\right)^{-1} F^{\prime}\left(x_{0}\right)\right\| \leq \frac{1}{1-b\left\|x_{k}-x_{0}\right\|} \leq \frac{1}{1-b r_{k}} \leq-\frac{1}{p^{\prime}\left(r_{k}\right)}=\frac{p^{\prime}\left(r_{0}\right)}{p^{\prime}\left(r_{k}\right)}$.

$\left(I I_{k}\right)\left\|\Gamma_{0} F\left(x_{k}\right)\right\| \leq \frac{b}{2}\left\|x_{k}-x_{k-1}\right\|^{2}+(1-\lambda)\left\|\Gamma_{0} F\left(x_{k-1}\right)\right\|$

$$
\begin{aligned}
& \leq \frac{b}{2}\left(r_{k}-r_{k-1}\right)^{2}-(1-\lambda) \frac{p\left(r_{k-1}\right)}{p^{\prime}\left(r_{0}\right)} \\
& =-\frac{1}{p^{\prime}\left(r_{0}\right)} \frac{b}{2}\left(r_{k}-r_{k-1}\right)^{2}-(1-\lambda) \frac{p\left(r_{k-1}\right)}{p^{\prime}\left(r_{0}\right)} \\
& =-\frac{1}{p^{\prime}\left(r_{0}\right)}\left[\frac{b}{2}\left(r_{k}-r_{k-1}\right)^{2}+(1-\lambda) p\left(r_{k-1}\right)\right]=-\frac{p\left(r_{k}\right)}{p^{\prime}\left(r_{0}\right)} .
\end{aligned}
$$

$\left(I I I_{k}\right)\left\|x_{k+1}-x_{k}\right\| \leq \lambda\left\|\Gamma_{k} F\left(x_{k}\right)\right\| \leq \lambda\left\|\Gamma_{k} F^{\prime}\left(x_{0}\right)\right\|\left\|\Gamma_{0} F\left(x_{k}\right)\right\|$

$$
\leq \lambda\left(\frac{p^{\prime}\left(r_{0}\right)}{p^{\prime}\left(r_{k}\right)}\right)\left(-\frac{p\left(r_{k}\right)}{p^{\prime}\left(r_{0}\right)}\right)=-\lambda \frac{p\left(r_{k}\right)}{p^{\prime}\left(r_{k}\right)}=r_{k+1}-r_{k}
$$

Now, to obtain the uniqueness region of the solution we assume that $\hat{x}$ is a solution located in the ball $B\left(x_{0}, r^{* *}\right)$, where $r^{* *}$ is defined in (11). Then:

$$
0=\Gamma_{0}\left[F\left(\hat{x}-F\left(x^{*}\right)\right]=W\left(\hat{x}-x^{*}\right),\right.
$$

where $W: X \rightarrow X$ is the lineal operator defined by:

$$
W x=\left[\int_{0}^{1} \Gamma_{0} F^{\prime}\left(x^{*}+t\left(\hat{x}-x^{*}\right)\right) d t\right] x, \quad x \in X .
$$

As $\left\|\hat{x}-x_{0}\right\|<r^{* *}$ and $\left\|x^{*}-x_{0}\right\| \leq r^{*}$, then:

$$
\begin{aligned}
\|I-W\| & =\left\|\int_{0}^{1} \Gamma_{0}\left[F^{\prime}\left(x^{*}+t\left(\hat{x}-x^{*}\right)\right)-F^{\prime}\left(x_{0}\right)\right] d t\right\| \\
& \leq \int_{0}^{1} b\left\|x^{*}+t\left(\hat{x}-x^{*}\right)-x_{0}\right\| d t \\
& \leq \int_{0}^{1} b\left((1-t)\left\|x^{*}-x_{0}\right\|+t\left\|\hat{x}-x_{0}\right\|\right) d t
\end{aligned}
$$




$$
<\frac{b}{2}\left(r^{*}+r^{* *}\right)=1
$$

Again by the Banach Lemma on inversion of operators (see [22]) there exists the inverse operator of $W$. Finally, taking into account (14), we deduce that $\hat{x}=x^{*}$. Hence, $x^{*}$ is the unique solution of $F(x)=0$ in the ball $B\left(x_{0}, r^{* *}\right)$. The proof of the theorem is complete.

Furthermore, we present the following corollary.

Corollary 4. If $h=1 / 2$ the convergence of the relaxed Newton's method to a root $x^{*}$ is also established. However, in this particular case, $t^{* *}=t^{*}=\frac{1}{b}$. That is the existence and uniqueness domain is the closed ball $\overline{B\left(x_{0}, \frac{1}{b}\right)}$.

Once we have proven the previous semilocal convergence results we can present the order of convergence of the method and error bounds. By the Schröder theorem, we can obtain the convergence order of the sequence (12). As $r_{n+1}=N_{\lambda, p}\left(r_{n}\right)$ where $N_{\lambda, p}\left(r_{n}\right)$ and $p(r)$ defined in (10) and (8), respectively:

$$
N_{\lambda, p}^{\prime}(x)=1-\lambda\left(-L_{p}(x)+1\right)=(1-\lambda)+\lambda L_{p}(x) .
$$

so we can ensure that:

$$
\begin{aligned}
r^{*}-r_{n+1} & =N_{\lambda, p}\left(r^{*}\right)-N_{\lambda, p}\left(r_{n}\right) \\
& =N_{\lambda, p}\left(r^{*}\right)-\left[N_{\lambda, p}\left(r^{*}\right)+N_{\lambda, p}^{\prime}\left(r^{*}\right)\left(r_{n}-r^{*}\right)+O\left(r_{n}-r^{*}\right)^{2}\right] \\
& =N_{\lambda, p}^{\prime}\left(r^{*}\right)\left(r^{*}-r_{n}\right)+O\left(r_{n}-r^{*}\right) .
\end{aligned}
$$

So linear convergence is proven, with asymptotic error constant $N_{\lambda, p}^{\prime}\left(r^{*}\right)=$ $(1-\lambda)$. Then the speed of convergence depends on the damping factor $\lambda$. This speed is greater when $\lambda$ is closer to 1 (see [21]).

Following a technique introduced by Ostrowski, we are going to present upper and lower bounds for the error.

Theorem 5. Under the assumptions of Theorem 3. Then, we have the following error bounds for the sequence $\left(x_{n}\right)$ given by the relaxed Newton's method:

$$
\frac{r^{*}\left(r^{* *}-r^{*}\right)(1-\lambda)^{n}}{r^{* *}-r^{*}(1-\lambda)^{n}} \leq\left\|x^{*}-x_{n}\right\| \leq \frac{r^{*}\left(r^{* *}-r^{*}\right) Q^{n}}{r^{* *}-r^{*} Q^{n}},
$$

where $Q=\frac{r^{*}+(1-\lambda) r^{* *}}{r^{* *}+(1-\lambda) r^{*}}, r^{*}$ and $r^{* *}$ are defined in (9) and (11), respectively.

Proof. Letting $a_{n}=r^{*}-r_{n}$ and $b_{n}=r^{* *}-r_{n}$. Then:

$a_{n+1}=r^{*}-r_{n+1}=r^{*}-r_{n}+\lambda \frac{p\left(r_{n}\right)}{p^{\prime}\left(r_{n}\right)}=a_{n}-\lambda \frac{a_{n} b_{n}}{a_{n}+b_{n}}=a_{n} \frac{a_{n}+(1-\lambda) b_{n}}{a_{n}+b_{n}}$,

and

$$
b_{n+1}=b_{n} \frac{b_{n}+(1-\lambda) a_{n}}{a_{n}+b_{n}} .
$$


And so, it's clear that

$$
\frac{a_{n+1}}{b_{n+1}}=\frac{a_{n}}{b_{n}} \frac{a_{n}+(1-\lambda) b_{n}}{b_{n}+(1-\lambda) a_{n}} .
$$

And so, we have the following:

$\frac{a_{n}+(1-\lambda) b_{n}}{b_{n}+(1-\lambda) a_{n}}=\frac{r^{*}-r_{n}+(1-\lambda)\left(r^{* *}-r_{n}\right)}{r^{* *}-r_{n}+(1-\lambda)\left(r^{*}-r_{n}\right)}=\frac{r^{*}+(1-\lambda) r^{* *}-r_{n}(2-\lambda)}{r^{* *}+(1-\lambda) r^{*}-r_{n}(2-\lambda)}$.

Now letting $f(x)=\frac{A-B x}{C-B x}$, and derivating:

$$
f^{\prime}(x)=\frac{-B(C-B x)+B(A-B x)}{(C-B x)^{2}}=\frac{B(A-C)}{(C-B x)^{2}} .
$$

In our particular case:

$$
\begin{aligned}
A-C & =r^{*}+(1-\lambda) r^{* *}-r^{* *}-(1-\lambda) r^{*} \\
& =\left(r^{*}-r^{* *}\right)+(1-\lambda)\left(r^{* *}-r^{*}\right)=-\lambda\left(r^{* *}-r^{*}\right)<0 .
\end{aligned}
$$

Then, as $0 \leq r_{n}<r^{*}$ :

$$
f(0) \geq f\left(r_{n}\right) \geq f\left(r^{*}\right) .
$$

Letting $q_{n}=\frac{a_{n}}{b_{n}}$, we compute the upper bound as:

$$
q_{n+1} \leq q_{n} \frac{r^{*}+(1-\lambda) r^{* *}}{r^{* *}+(1-\lambda) r^{*}}
$$

and setting $Q=\frac{r^{*}+(1-\lambda) r^{* *}}{r^{* *}+(1-\lambda) r^{*}}$, we have:

$$
q_{n+1} \leq Q q_{n} \leq Q^{2} q_{n-1} \leq \cdots \leq Q^{n+1} q_{0} .
$$

Taking into account, $q_{n} \leq Q^{n} q_{0}$, then:

$$
a_{n} \leq q_{0} b_{n} Q^{n}=q_{0}\left(r^{* *}-r_{n}\right) Q^{n}=q_{0}\left(r^{* *}-r_{n}\right) Q^{n}=q_{0}\left(r^{* *}-r^{*}+a_{n}\right) Q^{n} \text {. }
$$

In addition, we have $\left[1-q_{0} Q^{n}\right] a_{n} \leq q_{0}\left(r^{* *}-r^{*}\right) Q^{n}$ :

$$
a_{n} \leq \frac{q_{0}\left(r^{* *}-r^{*}\right) Q^{n}}{1-q_{0} Q^{n}} .
$$

On the other hand as $Q<1$, as $r^{*}+r^{* *}-\lambda r^{* *}<r^{*}-\lambda r^{*}+r^{* *}$, we can conclude that:

$$
a_{n} \leq \frac{r^{*}\left(r^{* *}-r^{*}\right) Q^{n}}{r^{* *}-r^{*} Q^{n}}
$$

Let's compute the lower bound:

$$
\begin{aligned}
q_{n+1} & \geq q_{n} \frac{r^{*}+(1-\lambda) r^{* *}-(2-\lambda) r^{*}}{r^{* *}+(1-\lambda) r^{*}-(2-\lambda) r^{*}}=q_{n} \frac{r^{*}(\lambda-1)+(1-\lambda) r^{* *}}{r^{* *}-r^{*}} \\
& =q_{n} \frac{(1-\lambda)\left(r^{* *}-r^{*}\right)}{r^{* *}-r^{*}}=(1-\lambda) q_{n} \geq \cdots \geq(1-\lambda)^{n+1} q_{0} .
\end{aligned}
$$

As $q_{n} \geq(1-\lambda)^{n} q_{0}$, then: $a_{n} \geq(1-\lambda)^{n} b_{n} q_{0}=(1-\lambda)^{n}\left(r^{* *}-r^{*}+r^{*}-r_{n}\right) q_{0}=(1-\lambda)^{n}\left(r^{* *}-r^{*}+a_{n}\right) q_{0}$. 
Consequently as $\left[1-q_{0}(1-\lambda)^{n}\right] a_{n} \geq\left(r^{* *}-r^{*}\right) q_{0}(1-\lambda)^{n}$ :

$$
a_{n} \geq \frac{r^{*}\left(r^{* *}-r^{*}\right)(1-\lambda)^{n}}{r^{* *}-r^{*}(1-\lambda)^{n}} .
$$

This ends the proof.

On the other hand,

$$
Q=Q(\lambda)=\frac{r^{*}+(1-\lambda) r^{* *}}{r^{* *}+(1-\lambda) r^{*}}=\frac{1-\frac{r^{* *}}{r^{*}+r^{* *}} \lambda}{1-\frac{r^{*}}{r^{*}+r^{* *}} \lambda} .
$$

Letting $A=\frac{r^{* *}}{r^{*}+r^{* *}}$ and $B=\frac{r^{*}}{r^{*}+r^{* *}}$,

$$
Q(\lambda)=\frac{1-A \lambda}{1-B \lambda}
$$

and so:

$$
Q^{\prime}(\lambda)=\frac{B-A}{(1-B \lambda)^{2}},
$$

and by the way of $B-A=\frac{r^{*}-r^{* *}}{r^{*}+r^{* *}}<0$, then function $Q(\lambda)$ is decreasing, and consequently the greater the damping factor is the faster method converges to the solution.

Lastly, we have the following corollary:

Corollary 6. If $\lambda=1$, from the equation (15) we have that $\frac{a_{n+1}}{b_{n+1}}=\left(\frac{a_{n}}{b_{n}}\right)^{2}$ and so the method has quadratic convergence (see [21]).

\section{Semilocal convergence under center-Lipschitz and Lipschitz conditions}

In this section we present a different semilocal convergence analysis for the relaxed Newton's method based on the center-Lipschitz and Lipschitz condition. First, we need a result on a scalar sequence which will be shown to be majorizing for $\left\{x_{n}\right\}$. The convergence of the sequences in this section is established for $\lambda \in(0,2)$. Notice that in Section 3 the convergence was established for $\lambda \in(0,1)$.

Lemma 2. Let $a>0, \beta>0, b>0$ and $\lambda \in(0,2)$ be given parameters. Set $M=|1-\lambda|$ and $\gamma=\frac{\beta}{b}$. Define parameter $\alpha$ by

$$
\alpha=\frac{2(\lambda-2 M \gamma)}{\lambda+\sqrt{\lambda^{2}+8 \gamma(\lambda-2 M \gamma)}} .
$$

Suppose that for $\delta=a b \lambda \gamma$ the following conditions are true:

$$
2 M \gamma<\lambda
$$$$
(\lambda-2) \delta^{2}+2(2+M) \delta-2<0,
$$ 


$$
\left(\frac{\lambda^{2}}{\delta}-4 M-4 \alpha+2 \alpha \lambda\right) \delta^{2}+2\left(\frac{M}{\gamma} \lambda+4 \alpha+2 \alpha M\right) \delta+4(M-\alpha) \leq 0,
$$

and

(20) $(M+\alpha)(\lambda-2(1-\alpha)) \delta^{2}+2(M+(3-2 \alpha) \alpha) \delta+2(1-\alpha)(M-\alpha) \leq 0$.

Then, scalar sequence $\left\{t_{n}\right\}$ defined by

$$
\left\{\begin{array}{l}
t_{0}=0, t_{1}=\lambda a, t_{2}=t_{1}+\frac{1}{2\left(1-\gamma b t_{1}\right)}\left[\lambda \gamma b\left(t_{1}-t_{0}\right)+2 M\right]\left(t_{1}-t_{0}\right) \\
t_{n+2}=t_{n+1}+\frac{1}{2\left(1-\gamma b t_{n+1}\right)}\left[\lambda b\left(t_{n+1}-t_{n}\right)+2 M\left(1+\gamma b t_{n}\right)\right]\left(t_{n+1}-t_{n}\right),
\end{array}\right.
$$

is well defined, increasing, bounded from above by

$$
t^{* *}=\left[1+\frac{\lambda \gamma b+2 M}{2(1-\delta)(1-\alpha)}\right] \lambda a
$$

and converges to its unique least upper bound $t^{*}$ which satisfies:

$$
t^{*} \in\left[t_{n}, t^{* *}\right] .
$$

Moreover, the following estimates hold for each $n=1,2, \ldots$

$$
0<t_{n+2}-t_{n+1} \leq \frac{\lambda \gamma b+2 M}{2(1-\delta)} \alpha^{n} n
$$

Proof. We first notice that $\alpha \in(0,1)$ by (16) and (17) and $M \in(0,1)$ since $\lambda \in(0,2)$. Let

$$
\alpha_{0}=\frac{\lambda b\left(t_{2}-t_{1}\right)+2\left(\gamma b t_{1}+1\right) M}{2\left(1-\gamma b t_{2}\right)} .
$$

Using (18) and (21) we get that

$$
\gamma b t_{2}<1
$$

Moreover, by (16), (19) and (26) we deduce that

$$
0<\alpha_{0} \leq \alpha .
$$

Next we shall show using induction on the integer $k=1,2, \ldots$ that

$$
0<\frac{\lambda b\left(t_{k+1}-t_{k}\right)+2 M\left(\gamma b t_{k}+1\right)}{2\left(1-\gamma b t_{2}\right)} \leq \alpha
$$

Estimate (28) is true for $k=1$ by (27). Then, using (21) for $n=1$ and (27) we obtain that

$$
\begin{aligned}
0<t_{3}-t_{2} \leq \alpha\left(t_{2}-t_{1}\right) \Rightarrow t_{3} & \leq t_{2}+\alpha\left(t_{2}-t_{1}\right) \\
& =t_{2}+(1+\alpha)\left(t_{2}-t_{1}\right)-\left(t_{2}-t_{1}\right) \\
t_{3} & \leq t_{1}+\frac{1-\alpha^{2}}{1-\alpha}\left(t_{2}-t_{1}\right)<t^{* *}
\end{aligned}
$$


Suppose that (28) holds for each $k=1,2, \ldots, m$. Then, we get that

$$
0<t_{k+2}-t_{k+1} \leq \alpha^{k}\left(t_{2}-t_{1}\right)
$$

and

$$
t_{k+2} \leq t_{1}+\frac{1-\alpha^{k+1}}{1-\alpha}\left(t_{2}-t_{1}\right)
$$

We must show that

$$
0 \leq \frac{\lambda b\left(t_{k+2}-t_{k+1}\right)+2 M\left(\gamma b t_{k}+1\right)}{2\left(1-\gamma b t_{k+1}\right)} \leq \alpha .
$$

Evidently, (32) holds, if

$$
\lambda b\left(t_{k+2}-t_{k+1}\right)+2\left(\gamma b t_{k}+1\right) M \leq 2 \alpha\left(1-\gamma b t_{k+1}\right)
$$

or using (30) and (31)

$$
\begin{aligned}
& \lambda b\left(t_{2}-t_{1}\right) \alpha^{k}+2 M \gamma b \frac{1-\alpha^{k}}{1-\alpha}\left(t_{2}-t_{1}\right)+2 \alpha \gamma b \frac{1-\alpha^{k+1}}{1-\alpha}\left(t_{2}-t_{1}\right) \\
& +2(\delta M+\alpha \delta+M-\alpha) \leq 0 .
\end{aligned}
$$

Estimate (33) motivates us to define recurrent functions $f_{k}$ on $(0,1)$ by

$$
\begin{aligned}
f_{k}(t)= & \lambda b\left(t_{2}-t_{1}\right) t^{k}+2 M \gamma b\left(t_{2}-t_{1}\right)\left(1+t+\cdots+t^{k-1}\right) \\
& +2 \gamma b\left(t_{2}-t_{1}\right) t\left(1+t+\cdots t^{k}\right)+2((M \gamma b+t \gamma b) \lambda a+M-t) .
\end{aligned}
$$

We need a relationship between two consecutive function $f_{k}$. Using (34) we get that:

$$
f_{k+1}(t)=f_{k+1}(t)-f_{k}(t)+f_{k}(t)=f_{k}(t)+g(t)\left(t_{2}-t_{1}\right) t^{k}
$$

where

$$
g(t)=2 \gamma b t^{2}+\lambda b t+(2 M \gamma-\lambda) b .
$$

It follows from (17) and (36) that polynomial $g$ has a unique positive root $\alpha$ given by (16). In view of (34), estimate (33) holds if

$$
f_{k}(\alpha) \leq 0 \text {. }
$$

Define function $f_{\infty}$ on $(0,1)$ by

$$
f_{\infty}(t)=\lim _{k \rightarrow \infty} f_{k}(t)
$$

We have from (35) and (36) that

$$
f_{k+1}(\alpha)=f_{k}(\alpha) .
$$

Then, it follows from (38) and (39) that (37) holds if

$$
f_{\infty}(\alpha) \leq 0 \text {. }
$$

By letting $k \rightarrow \infty$ and by (20) we have that (40) is true, since

$$
f_{\infty}(\alpha)=2\left[M \delta+\alpha \delta+M-\alpha(1-\gamma)+\frac{M \gamma b}{1-\alpha}\left(t_{2}-t_{1}\right)+\frac{\gamma b \alpha}{1-\alpha}\left(t_{2}-t_{1}\right)\right] \leq 0 .
$$


The induction for (28) is complete. Hence, the sequence $\left\{t_{n}\right\}$ is increasing, bounded from above by $t^{* *}$ given in $(22)$ and as such it converges to its unique least upper bound $t^{*}$ which satisfies $(23)$. The proof is complete.

Remark 2. Quadratic inequalities (18)-(20) describe the smallness of $a$ and can be solved for $a$. However, we decided to leave them as uncluttered as possible, since this representation is too long. It is simple algebra to show that in the interesting case when $\lambda=1$ (Newton's method) reduce to (see [6]):

$$
h_{*}=L a \leq 1 / 2 \text {, }
$$

where

$$
L=\frac{1}{8}\left(4 b_{0}+\sqrt{b_{0} b+8 b_{0}^{2}}+\sqrt{b_{0} b}\right) .
$$

If $b_{0}=b$, then (41) reduces to the famous for its simplicity and clarity NewtonKantorovich hypothesis given in condition (iv) of Theorem 3. Notice that

$$
h \leq 1 / 2 \Rightarrow h_{*} \leq 1 / 2
$$

but not necessarily viceversa unless if $b_{0}=0$ and $\frac{h_{*}}{h} \rightarrow 0$ as $\frac{b_{0}}{b} \rightarrow 0$. Hence, the applicability of Newton's method has been extended.

Let us introduce the notation for fixed $N=0,1, \ldots$

$$
\delta_{N}=\gamma b\left(t_{N+1}-t_{N}\right) .
$$

Note that $\delta_{0}=\delta$. Next, we present the following weaker than Lemma 2 result for the convergence of sequence $\left\{t_{n}\right\}$. The proof simply follows from the proof of Lemma 2 by replacing $\delta$ by $\delta_{N}$.

Lemma 3. Suppose that (17) and (18)-(20) hold with $\delta_{N}$ replacing $\delta$ for $\delta_{N}$ fixed $N=0,1, \ldots$. Moreover, suppose that

$$
0<t_{1}<t_{2}<\cdots<t_{N+1}<\frac{1}{\gamma b} .
$$

Then, scalar sequence $\left\{t_{n}\right\}$, given in (21) is well defined, increasing, bounded from above by

$$
t_{N}^{* *}=t_{N-1}+\frac{1}{1-\alpha}\left(t_{N}-t_{N-1}\right)
$$

for $N=1,2, \ldots$ and converges to its unique least upper bound $t_{N}^{*}$ satisfying

$$
t_{N+1} \leq t_{N}^{*} \leq t_{N}^{* *}
$$

Moreover, the following estimates hold for each $n=0,1, \ldots$

$$
0<t_{N+n}-t_{N+n-1} \leq \alpha^{n-1}\left(t_{N+1}-t_{N}\right) .
$$

Remark 3. If $N=0$, Lemma 3 reduces to Lemma 2. Clearly Lemma 3 is weaker than Lemma 2.

Next, we present the semilocal convergence of relaxed Newton's method using a center-Lipschitz and a Lipschitz condition as well as $\left\{t_{n}\right\}$ as the majorizing sequence for $\left\{x_{n}\right\}$. 
Theorem 7. Let $F: \Omega \subset X \rightarrow Y$ be Fréchet-differentiable. Suppose that there exist $x_{0} \in \Omega, \beta>0, b>0, a>0, \lambda \neq 0$ such that there exists $F^{\prime}\left(x_{0}\right)^{-1}$, in addition

$$
\begin{gathered}
\left\|F^{\prime}\left(x_{0}\right)^{-1} F\left(x_{0}\right)\right\| \leq a, \\
\left\|F^{\prime}\left(x_{0}\right)^{-1}\left(F^{\prime}(x)-F^{\prime}\left(x_{0}\right)\right)\right\| \leq \beta\left\|x-x_{0}\right\|, \quad \forall x \in \Omega, \\
\left\|F^{\prime}\left(x_{0}\right)^{-1}\left(F^{\prime}(x)-F^{\prime}(y)\right)\right\| \leq b\|x-y\|, \forall x, y \in \Omega, \\
\bar{B}\left(x_{0}, t^{*}\right) \subseteq \Omega
\end{gathered}
$$

and hypothesis of Lemma 2 or Lemma 3 hold. Then, sequence $\left\{x_{n}\right\}$ generated by the relaxed Newton's method is well defined, remains in $\bar{B}\left(x_{0}, t^{*}\right)$ for each $n=0,1, \ldots$ and converges to a solution $x^{*} \in \bar{B}\left(x_{0}, t^{*}\right)$ of equation $F(x)=0$. Moreover, the following estimates hold for each $n=0,1, \ldots$

$$
\left\|x_{n+1}-x_{n}\right\| \leq t_{n+1}-t_{n}
$$

and

$$
\left\|x_{n}-x^{*}\right\| \leq t_{n}-t^{*}
$$

where sequence $\left\{t_{n}\right\}$ is given in (21). Furthermore, if there exists $R \geq t^{*}$ such that

$$
\bar{B}\left(x_{0}, t^{*}\right) \subseteq D
$$

and

$$
\beta\left(t^{*}+R\right)<2,
$$

then, the solution $x^{*}$ is unique in $\bar{B}\left(x_{0}, R\right)$.

Proof. The proof follows in an analogous way as the proof of Theorem 3 but we use the Ostrowski decomposition

$$
\begin{aligned}
& F^{\prime}\left(x_{0}\right)^{-1} F\left(x_{n+1}\right) \\
= & F^{\prime}\left(x_{0}\right)^{-1} \int_{0}^{1}\left[F^{\prime}\left(x_{n}+t\left(x_{n+1}-x_{n}\right)\right)-F^{\prime}\left(x_{n}\right)\right]\left(x_{n+1}-x_{n}\right) d t \\
& +\left(1-\frac{1}{\lambda}\right)\left(I+F^{\prime}\left(x_{0}\right)^{-1}\left(F^{\prime}\left(x_{n}\right)-F^{\prime}\left(x_{0}\right)\right)\left(x_{n+1}-x_{n}\right)\right.
\end{aligned}
$$

instead of (13). For the estimates on upper bounds on the norms

$$
\left\|F^{\prime}\left(x_{n}\right)^{-1} F^{\prime}\left(x_{0}\right)\right\|
$$

and the uniqueness part we use the more precise and cheaper center-Lipschitz condition instead of the Lispchitz condition.

Remark 4. Note that the definition of $t_{2}$ (see $(21)$ ) is justified from the Ostroswki representation (43) for $n=0$, since then the center-Lipschitz (and not the Lipschitz) condition is used to obtain the estimate on

$\left\|F^{\prime}\left(x_{0}\right)^{-1}\right\| \int_{0}^{1}\left[F^{\prime}\left(x_{0}+t\left(x_{1}-x_{0}\right)\right)-F^{\prime}\left(x_{0}\right)\right] d t\left\|\leq \frac{\beta}{2}\right\| x_{1}-x_{0} \|^{2} \leq \frac{\beta}{2}\left(t_{1}-t_{0}\right)^{2}$ 
instead of

$$
\left\|F^{\prime}\left(x_{0}\right)^{-1}\right\| \int_{0}^{1}\left[F^{\prime}\left(x_{0}+t\left(x_{1}-x_{0}\right)\right)-F^{\prime}\left(x_{0}\right)\right] d t\left\|\leq \frac{b}{2}\right\| x_{1}-x_{0} \|^{2} .
$$

We complete this section with another result for the relaxed Newton's method with simpler convergence conditions than in Theorem 7 .

Theorem 8. Let $F: \Omega \subset X \rightarrow Y$ be Fréchet-differentiable. Suppose that there exist $x_{0} \in \Omega, \beta>0, b>0, a>0, \lambda \in(0,2)$ such that there exists $F^{\prime}\left(x_{0}\right)^{-1}$, in addition

$$
\begin{gathered}
\left\|F^{\prime}\left(x_{0}\right)^{-1} F\left(x_{0}\right)\right\| \leq a, \\
\left\|F^{\prime}\left(x_{0}\right)^{-1}\left(F^{\prime}(x)-F^{\prime}\left(x_{0}\right)\right)\right\| \leq \beta\left\|x-x_{0}\right\|, \quad \forall x \in \Omega, \\
\left\|F^{\prime}\left(x_{0}\right)^{-1}\left(F^{\prime}(x)-F^{\prime}(y)\right)\right\| \leq b\|x-y\|, \quad \forall x, y \in \Omega .
\end{gathered}
$$

Moreover, suppose that

$$
h_{1}=\lambda a b \max \{\lambda, \gamma(1+M)\} \leq \frac{1}{2}(1-M)^{2},
$$

and

$$
\bar{B}\left(x_{0}, s^{*}\right) \subseteq \Omega
$$

where $M$ and $\gamma$ are given in Lemma 2 and

$$
\begin{gathered}
s^{*}=\frac{1-M-\sqrt{(1-M)^{2}-2 h_{1}}}{\sigma}, \\
\sigma=b \max \{\lambda, \gamma(1+M)\} .
\end{gathered}
$$

Then, sequence $\left\{x_{n}\right\}$ generated by the relaxed Newton's method is well defined, remains in $\bar{B}\left(x_{0}, s^{*}\right)$ for all $n \geq 0$ and converges to a solution $x^{*} \in \bar{B}\left(x_{0}, s^{*}\right)$ of equation $F(x)=0$. Moreover the equation $F(x)=0$, has a unique solution $x^{*} \in S$, where

$$
S=\left\{\begin{array}{l}
\bar{B}\left(x_{0}, s^{*}\right) \bigcap \Omega \text { if } h_{1}=\frac{1}{2}(1-M)^{2}, \\
\bar{B}\left(x_{0}, s^{* *}\right) \bigcap \Omega \text { if } h_{1}<\frac{1}{2}(1-M)^{2}
\end{array}\right.
$$

and

$$
s^{* *}=\frac{1-M+\sqrt{(1-M)^{2}-2 h_{1}}}{\sigma} .
$$

Furthermore, the following estimates hold for each $n=0,1, \ldots$

$$
\left\|x_{n+1}-x_{n}\right\| \leq s_{n+1}-s_{n}
$$

and

$$
\left\|x_{n}-x^{*}\right\| \leq s^{*}-s_{n},
$$

where majorizing sequence $\left\{s_{n}\right\}$ is defined by

where

$$
s_{0}=0, s_{n+1}=s_{n}+\frac{f\left(s_{n}\right)}{g\left(s_{n}\right)},
$$

$$
f(t)=\frac{\sigma}{2} t^{2}-(1-M) t+\lambda a
$$


and

$$
g(t)=1-\gamma b t
$$

Proof. It follows easily from $2 \sigma \lambda a \leq(1-M)^{2}$ that the function $f(t)$ has two positive roots $s^{*}$ and $s^{* *}, s^{*} \leq s^{* *}$ and $s_{n} \leq s_{n+1}$, so that the sequence $\left\{s_{n}\right\}$ converges to $s^{*}$. As in Theorem 7 we obtain $F^{\prime}\left(x_{n}\right)^{-1} \in L(Y, X)$,

$$
\left\|F^{\prime}\left(x_{n}\right)^{-1} F^{\prime}\left(x_{0}\right)\right\| \leq \frac{1}{1-\beta\left\|x_{n}-x_{0}\right\|} \leq \frac{1}{1-\beta s_{n}} \leq \frac{1}{1-b \gamma s_{n}} \leq \frac{1}{g\left(s_{n}\right)}
$$

and

$$
\begin{aligned}
& \left\|x_{n+1}-x_{n}\right\| \\
\leq & \frac{1}{g\left(s_{n}\right)}\left[\frac{\lambda b}{2}\left\|x_{n}-x_{n-1}\right\|+\left(M \gamma b\left\|x_{n}-x_{0}\right\|+M\right)\right]\left\|x_{n}-x_{n-1}\right\| \\
\leq & \frac{1}{g\left(s_{n}\right)}\left(\frac{\sigma}{2}\left(s_{n}-s_{n-1}\right)+M\left(\gamma b s_{n-1}+1\right)\right)\left(s_{n}-s_{n-1}\right) \\
= & \frac{1}{g\left(s_{n}\right)}\left(\frac{\sigma}{2}\left(s_{n}-s_{n-1}\right)^{2}+M \gamma b\left(s_{n}-s_{n-1}\right) s_{n-1}\right) \\
& +\frac{1}{g\left(s_{n}\right)}\left(M\left(s_{n}-s_{n-1}\right)-g\left(s_{n-1}\right)\left(s_{n}-s_{n-1}\right)+f\left(s_{n-1}\right)\right) \\
= & \frac{1}{g\left(s_{n}\right)}\left(\frac{\sigma}{2} s_{n}^{2}-(1-M) s_{n}+\lambda a-(\sigma-M \gamma b-\gamma b) s_{n-1}\left(s_{n}-s_{n-1}\right)\right) \\
\leq & \frac{f\left(s_{n}\right)}{g\left(s_{n}\right)}=s_{n+1}-s_{n} .
\end{aligned}
$$

Hence, we have for any $n$

$\left\|x_{n+1}-x_{n}\right\| \leq s_{n+1}-s_{n}$,

$\left\|F^{\prime}\left(x_{0}\right)^{-1}\left(F^{\prime}\left(x_{n+1}\right)-F^{\prime}\left(x_{0}\right)\right)\right\| \leq \beta\left\|x_{n+1}-x_{0}\right\| \leq \beta s_{n+1} \leq \gamma b s_{n+1} \leq \gamma b s^{*}<1$ and

$$
\left\|x_{n}-x_{0}\right\| \leq s_{n} \leq s^{*}
$$

It follows that: $x_{n} \in \bar{B}\left(x_{0}, s^{*}\right)$, sequence $\left\{x_{n}\right\}$ is complete in a Banach space $X$ and as such it converges to some $x^{*} \in \bar{B}\left(x_{0}, s^{*}\right)$ (since $\bar{B}\left(x_{0}, s^{*}\right)$ is a closed set). We also have that

$$
\begin{aligned}
\left\|F^{\prime}\left(x_{0}\right)^{-1} F\left(x_{n}\right)\right\| & \leq\left(\frac{\sigma}{2}\left(s_{n}-s_{n-1}\right)+M\left(\gamma b s_{n}+1\right)\right)\left(s_{n}-s_{n-1}\right) \\
& \leq\left(\frac{\sigma}{2}\left(s_{n}-s_{n-1}\right)+M\left(\gamma b s^{*}+1\right)\right)\left(s_{n}-s_{n-1}\right) \rightarrow 0,
\end{aligned}
$$

as $n \rightarrow \infty$, so that $F\left(x^{*}\right)=0$. Estimate (46) follows from (45) by using standard majorizing techniques (see [6], [7]). Finally, for the uniqueness part see the more general proof of Theorem 9 in Section 5. The proof of the theorem is complete.

Remark 5. The following two conditions are true. 
(a) Hypothesis $h_{1} \leq \frac{1}{2}(1-M)^{2}$ for $0<\lambda \leq 1$ reduces to

$$
h_{1}=a b \frac{\max \{\lambda, \gamma(2-\lambda)\}}{\lambda} \leq \frac{1}{2}
$$

which is not weaker than $h=a b \leq 1 / 2$. However, hypothesis $h=$ $a b \leq 1 / 2$ cannot be used as the sufficient convergence condition for the relaxed Newton's method for $\lambda \in(1,2)$. In practice we shall test all the " $\mathrm{h}$ " hypotheses introduced in this study to see if any of them is satisfied.

(b) Results analogous to Lemma 3 can now follow by exchanging sequence $\left\{t_{n}\right\}$ by $\left\{s_{n}\right\}$.

\section{Extended semilocal convergence}

In this section we present a semilocal convergence result to solve a nonlinear equation of the form:

$$
F(x)+G(x)=0,
$$

where $F$ is defined in the introduction and $G: \Omega \rightarrow Y$ is continuous. Then, the relaxed Newton's method for generating a sequence approximating $x^{*}$ is defined by:

$$
x_{n+1}=x_{n}-\lambda F^{\prime}\left(x_{n}\right)^{-1}(F(x)+G(x)), n \geq 0 .
$$

Clearly if $G=0$ on $\Omega$, then method (48) reduces to method (6). In this paper we used three different techniques for our semilocal convergence results. However, for the method (48) we shall only use the technique of Theorem 9. For brevity, we leave the analysis of the other two techniques to the motivated reader.

Theorem 9. Let $F: \Omega \subset X \rightarrow Y$ be Fréchet-differentiable and $G: \Omega \rightarrow Y$ be continuous. Suppose that there exist $x_{0} \in \Omega, \beta>0, b>0, a>0, K \geq 0$, $\lambda \in(0,2)$ such that there exists $F^{\prime}\left(x_{0}\right)^{-1}$, in addition

$$
\begin{aligned}
\| F^{\prime}\left(x_{0}\right)^{-1}\left(F\left(x_{0}\right)+G\left(x_{0}\right) \|\right. & \leq a, \\
\left\|F^{\prime}\left(x_{0}\right)^{-1}\left(F^{\prime}(x)-F^{\prime}\left(x_{0}\right)\right)\right\| & \leq \beta\left\|x-x_{0}\right\|, \quad \forall x \in \Omega, \\
\left\|F^{\prime}\left(x_{0}\right)^{-1}\left(F^{\prime}(x)-F^{\prime}(y)\right)\right\| & \leq b\|x-y\|, \quad \forall x, y \in \Omega, \\
\left\|F^{\prime}\left(x_{0}\right)^{-1}(G(x)-G(y))\right\| & \leq K\|x-y\|, \quad \forall x, y \in \Omega .
\end{aligned}
$$

Moreover, suppose

$$
\begin{gathered}
\mu=M+\lambda K<1, \\
h_{2}=\lambda a b \max \{\lambda, \gamma(1+M)\} \leq 1 / 2(1-\mu)^{2},
\end{gathered}
$$

and

$$
\bar{B}\left(x_{0}, s^{*}\right) \subseteq \Omega,
$$


where $M$ and $\gamma$ are given in Lemma 2 and

$$
\begin{aligned}
s^{*} & =\frac{1-\mu-\sqrt{(1-\mu)^{2}-2 h_{2}}}{\sigma} \\
\sigma & =b \max \{\lambda, \gamma(1+M)\} .
\end{aligned}
$$

Then the sequence $\left\{x_{n}\right\}$ generated by the relaxed Newton's method (48) is well defined, remains in $\bar{B}\left(x_{0}, s^{*}\right)$ for all $n \geq 0$ and converges to a solution $x^{*} \in$ $\bar{B}\left(x_{0}, s^{*}\right)$ of equation $F(x)+G(x)=0$. Moreover, the equation $F(x)+G(x)=0$ has a unique solution $x^{*} \in S$, where

$$
S=\left\{\begin{array}{l}
\bar{B}\left(x_{0}, s^{*}\right) \bigcap \Omega \quad \text { if } h_{2}=\frac{1}{2}(1-\mu)^{2}, \\
\bar{B}\left(x_{0}, s^{* *}\right) \bigcap \Omega \text { if } h_{2}<\frac{1}{2}(1-\mu)^{2},
\end{array}\right.
$$

and

$$
s^{* *}=\frac{1-\mu+\sqrt{(1-\mu)^{2}-2 h_{2}}}{\sigma} .
$$

Furthermore, the following estimates hold for each $n=0,1, \ldots$

$$
\left\|x_{n+1}-x_{n}\right\| \leq s_{n+1}-s_{n}
$$

and

$$
\left\|x_{n}-x^{*}\right\| \leq s^{*}-s_{n},
$$

where majorizing sequence $\left\{s_{n}\right\}$ is defined by

$$
s_{0}=0, s_{n+1}=s_{n}+\frac{f\left(s_{n}\right)}{g\left(s_{n}\right)},
$$

where

$$
f(t)=\frac{\sigma}{2} t^{2}-(1-\mu) t+\lambda a
$$

and

$$
g(t)=1-\gamma b t
$$

Proof. Using (48) we introduce as in Theorem 7 the Ostrowski decomposition to obtain

$$
\begin{aligned}
& F^{\prime}\left(x_{0}\right)^{-1}\left(F\left(x_{n+1}\right)+G\left(x_{n+1}\right)\right) \\
= & F^{\prime}\left(x_{0}\right)^{-1} \int_{0}^{1}\left[F^{\prime}\left(x_{n}+t\left(x_{n+1}-x_{n}\right)\right)-F^{\prime}\left(x_{n}\right)\right]\left(x_{n+1}-x_{n}\right) d t \\
& +\left(1-\frac{1}{\lambda}\right)\left(I+F^{\prime}\left(x_{0}\right)^{-1}\left(F^{\prime}\left(x_{n}\right)-F^{\prime}\left(x_{0}\right)\right)\left(x_{n+1}-x_{n}\right)\right. \\
& +F^{\prime}\left(x_{0}\right)^{-1}\left(G\left(x_{n+1}\right)-G\left(x_{n}\right)\right) .
\end{aligned}
$$

Then, following the proof of Theorem 8 , we obtain in turn that

$$
\begin{aligned}
& \left\|x_{n+1}-x_{n}\right\| \\
\leq & \frac{1}{g\left(s_{n}\right)}\left[\frac{\lambda b}{2}\left\|x_{n}-x_{n-1}\right\|+M \gamma b\left\|x_{n}-x_{0}\right\|+\mu\right]\left\|x_{n}-x_{n-1}\right\|
\end{aligned}
$$




$$
\begin{aligned}
& \leq \frac{1}{g\left(s_{n}\right)}\left(\frac{\sigma}{2}\left(s_{n}-s_{n-1}\right)^{2}+M \gamma b s_{n-1}+\mu\right)\left(s_{n}-s_{n-1}\right) \\
& \leq \frac{1}{g\left(s_{n}\right)}\left(\frac{\sigma}{2}\left(s_{n}-s_{n-1}\right)^{2}+M\left(s_{n}-s_{n-1}\right) s_{n-1}+\mu\left(s_{n}-s_{n-1}\right)\right) \\
& \quad \quad-\frac{1}{g\left(s_{n}\right)}\left(g\left(s_{n-1}\right)\left(s_{n}-s_{n-1}\right)-f\left(s_{n-1}\right)\right) \\
& =\frac{1}{g\left(s_{n}\right)}\left(\frac{\sigma}{2} s_{n}^{2}-(1-\mu) s_{n}+\lambda a-(\sigma-M \gamma b-\gamma b) s_{n-1}\left(s_{n}-s_{n-1}\right)\right) \\
& \leq \frac{f\left(s_{n}\right)}{g\left(s_{n}\right)}=s_{n+1}-s_{n} .
\end{aligned}
$$

The rest follows as in Theorem 8 until

$$
\left\|F^{\prime}\left(x_{0}\right)^{-1}\left(F\left(x_{n}\right)+G\left(x_{n}\right)\right)\right\| \leq\left(\frac{\sigma}{2}\left(s_{n}-s_{n-1}\right)^{2}+M \gamma b s^{*}+\mu\right)\left(s_{n}-s_{n-1}\right) \rightarrow 0
$$

as $n \rightarrow \infty$. Hence, $F\left(x^{*}\right)+G\left(x^{*}\right)=0$. Finally, in order for us to show the uniqueness part, let us assume that $F\left(y^{*}\right)+G\left(y^{*}\right)=0$ for some $y^{*} \in \Omega$. Using (48) we obtain the identity

$$
\begin{aligned}
y^{*}-x_{n}= & F^{\prime}\left(x_{0}\right)^{-1} \lambda \int_{0}^{1}\left[F^{\prime}\left(y^{*}+t\left(x_{n}-y^{*}\right)\right)-F^{\prime}\left(x_{n}\right)\right]\left(x_{n}-y^{*}\right) d t \\
& +(1-\lambda)\left(I+F^{\prime}\left(x_{0}\right)^{-1}\left(F^{\prime}\left(x_{n}\right)-F^{\prime}\left(x_{0}\right)\right)\right)\left(x_{n}-y^{*}\right) \\
& +\lambda F^{\prime}\left(x_{0}\right)^{-1}\left(G\left(x_{n}\right)-G\left(y^{*}\right)\right) .
\end{aligned}
$$

Then, for $n=0$ we get

$$
\left\|y^{*}-x_{1}\right\| \leq\left(\frac{\sigma}{2}\left\|y^{*}-x_{0}\right\|+\mu\right)\left\|y^{*}-x_{0}\right\|=\varphi(\xi),
$$

where $\xi=\left\|y^{*}-x_{0}\right\|$. We have that $\left\|y^{*}-x_{0}\right\| \leq\left\|y^{*}-x_{1}\right\|+\left\|x_{1}-x_{0}\right\| \leq \varphi(\xi)+\lambda a$. That if $f(\xi) \geq 0$. Hence, $y^{*} \in \bar{B}\left(x_{0}, s^{*}\right)$. Using induction we shall show that

$$
\left\|y^{*}-x_{k}\right\| \leq s^{*}-s_{n}, n \geq 0 .
$$

The preceding estimate is true for $k=0$, since $y^{*} \in \bar{B}\left(x_{0}, s^{*}\right)$. Let $\left\|y^{*}-x_{k}\right\| \leq$ $s^{*}-s_{k}$. Then, we have in turn that

$$
\begin{aligned}
& \left\|y^{*}-x_{k}\right\| \\
\leq & \frac{1}{g\left(s_{k}\right)}\left[\frac{\sigma}{2}\left\|y^{*}-x_{k}\right\|+M\left\|x_{k}-x_{0}\right\|+\mu\right]\left\|y^{*}-x_{k}\right\| \\
\leq & \frac{1}{g\left(s_{k}\right)}\left[\frac{\sigma}{2} s^{* 2}+\mu s^{*}-(\sigma-M \gamma b) s_{k}\left(s^{*}-s_{k}\right)-\frac{\sigma}{2} s_{k}^{2}-\mu s_{k}\right] \\
= & \frac{1}{g\left(s_{k}\right)}\left[s^{*}-\lambda a-(\sigma-M \gamma b) s_{k}\left(s^{*}-s_{k}\right)-\frac{\sigma}{2} s_{k}^{2}-\mu s_{k}\right] \\
= & s^{*}-s_{k}+\frac{1}{g\left(s_{k}\right)}\left[-\left(s^{*}-s_{k}\right) g\left(s_{k}\right)+s^{*}-\lambda a-\frac{\sigma}{2} s_{k}^{2}-\mu s_{k}\right] \\
& -\frac{1}{g\left(s_{k}\right)}\left[(\sigma-M \gamma b) s_{k}\left(s^{*}-s_{k}\right)\right]
\end{aligned}
$$




$$
\begin{aligned}
& =s^{*}-s_{k}-\frac{1}{g\left(s_{k}\right)}\left[\frac{\sigma}{2} s_{k}^{2}-(1-\mu) s_{k}+\lambda a+(\sigma-M \gamma b-\gamma b) s_{k}\left(s^{*}-s_{k}\right)\right] \\
& \leq s^{*}-s_{k+1} .
\end{aligned}
$$

That is, we have that $\lim _{k \rightarrow \infty} x_{k}=y^{*}$. But, we showed $\lim _{k \rightarrow \infty} x_{k}=x^{*}$. Hence, we deduce that $x^{*}=y^{*}$. The proof of the theorem is complete.

\section{Numerical examples and applications}

Example 1. Extended semilocal convergence. Consider the following nonlinear integral equation of mixed Hammerstein type

(52) $x(s)=f(s)+\int_{A}^{B} G(s, t) \alpha(x(t)-f(t))^{2}+\beta|x(t)-f(t)| d t, s \in[A, B]$,

where $x, f \in C[A, B], \alpha, \beta \in \mathbb{R}$ and the kernel $G$ is the Green function

$$
G(s, t)= \begin{cases}\frac{(B-s)(t-A)}{B-A}, & t \leq s, \\ \frac{(s-A)(B-t)}{B-A}, & s \leq t .\end{cases}
$$

To simplify the analysis we choose $A=0, B=1, \alpha=\beta=1 / 2$ and $f(s)=0$. To solve $(52)$, we transform it into a finite dimensional problem by using a process of discretization. For this, we approximate the integral that appears in (52) by the Gauss-Legendre formula

$$
\int_{0}^{1} h(t) d t \simeq \sum_{i=1}^{8} w_{i} h\left(t_{i}\right)
$$

where the nodes $t_{i}$ and the weights $w_{i}$ are known.

If we denote the approximation of $x\left(t_{i}\right)$ by $x_{i}(i=1,2, \ldots, 8)$, then $(52)$ is equivalent to the following nonlinear system of equations

$$
x_{i}=\frac{1}{2} \sum_{j=1}^{8} a_{i j}\left(x_{j}^{2}+\left|x_{j}\right|\right), \quad j=1,2, \ldots, 8,
$$

where

$$
a_{i j}= \begin{cases}w_{j} t_{j}\left(1-t_{i}\right) & \text { if } j \leq i \\ w_{j} t_{i}\left(1-t_{j}\right) & \text { if } j>i\end{cases}
$$

System (53) is now written as $F(\mathbf{x})+G(\mathbf{x})=\mathbf{0}$ where

$$
F(\mathbf{x}) \equiv \mathbf{x}-\frac{1}{2} A \mathbf{v}_{\mathbf{x}}, \quad G(\mathbf{x}) \equiv \frac{1}{2} A \mathbf{w}_{\mathbf{x}} \quad F: \mathbb{R}^{8} \longrightarrow \mathbb{R}^{8},
$$

where

$$
\begin{gathered}
\mathbf{x}=\left(x_{1}, x_{2}, \ldots, x_{8}\right)^{T}, A=\left(a_{i j}\right)_{i, j=1}^{8}, \mathbf{v}_{\mathbf{x}}=\left(x_{1}^{2}, x_{2}^{2}, \ldots, x_{8}^{2}\right)^{T}, \\
\mathbf{w}_{\mathbf{x}}=\left(\left|x_{1}\right|,\left|x_{2}\right|, \ldots,\left|x_{8}\right|\right)^{T} .
\end{gathered}
$$

Moreover, $F^{\prime}(\mathbf{x})=I-A D(\mathbf{x})$, where $D(\mathbf{x})=\operatorname{diag}\left\{x_{1}, x_{2}, \ldots, x_{8}\right\}$.

Choosing as starting point $\mathbf{x}_{\mathbf{0}}=(1,1, \ldots, 1)^{T}$ and the max-norm, we obtain $a=1.13821 \cdots, b=\beta=0.140636 \cdots, k=0.070318 \cdots$. If we take for instance 
$\lambda=0.9$ it follows $\mu=M+\lambda k=0.535159 \cdots<1$ and $h_{2}=0.0400186 \cdots$. In consequence, by Theorem 9 , the relaxed Newton's method with $\lambda=0.9$ converges to the trivial solution $\mathbf{x}^{*}=(0,0, \ldots, 0)^{T}$ of system $(53)$. The existence of the solution is guaranteed in $\overline{B\left(\mathbf{x}_{\mathbf{0}}, 1.36529 \cdots\right)}$ and the uniqueness in $B\left(\mathbf{x}_{\mathbf{0}}, 11.8558 \cdots\right)$.

Moreover, in Table 1 we can establish the following estimates of the error $\left\|\mathbf{x}_{\mathbf{n}}-\mathbf{x}^{*}\right\|$ that show in the following table.

TABLE 1. Estimates of the error $\left\|\mathbf{x}_{\mathbf{n}}-\mathbf{x}^{*}\right\|$ applying the relaxed Newton's method with $\lambda=0.9$ and $\lambda=1$ (Newton's method), to solve system (53)

\begin{tabular}{|c|c||l|}
\hline \hline$n$ & $\lambda=0.9,\left\|\mathbf{x}_{\mathbf{n}}-\mathrm{x}^{*}\right\|$ & $\lambda=1,\left\|\mathbf{x}_{\mathbf{n}}-\mathbf{x}^{*}\right\|$ \\
\hline 1 & $3.409021 \cdots \times 10^{-1}$ & $2.270807 \cdots \times 10^{-1}$ \\
2 & $6.788876 \cdots \times 10^{-2}$ & $2.332819 \cdots \times 10^{-2}$ \\
3 & $1.240100 \cdots \times 10^{-2}$ & $2.069182 \cdots \times 10^{-3}$ \\
4 & $2.221346 \cdots \times 10^{-3}$ & $1.803860 \cdots \times 10^{-4}$ \\
5 & $3.964426 \cdots \times 10^{-4}$ & $1.570110 \cdots \times 10^{-5}$ \\
6 & $7.0706150 \cdots \times 10^{-5}$ & $1.366465 \cdots \times 10^{-6}$ \\
7 & $1.2609058 \cdots \times 10^{-5}$ & $1.189218 \cdots \times 10^{-7}$ \\
8 & $2.2485316 \cdots \times 10^{-6}$ & $1.034962 \cdots \times 10^{-8}$ \\
\hline \hline
\end{tabular}

Example 2. Semilocal convergence under center-Lipschitz and Lipschitz conditions. In the following example, we consider the real function

$$
x^{3}-0.49=0 \text {. }
$$

We take the starting point $x_{0}=1$ and we consider the domain $\Omega=B\left(x_{0}, 1\right)$. In this case, we obtain

$$
\begin{aligned}
& a=0.17, \\
& b=3.02, \\
& \beta=2.51
\end{aligned}
$$

and

$$
\gamma=0.831126 \cdots
$$

Notice that Kantorovich hypothesis $h \leq 0.5$ is not satisfied. Now, taking $\lambda=$ 0.99 , we obtain that for $\delta_{1}=0.160253 \cdots$, hypotheses of Lemma 3 hold. Hence, conditions of convergence for the relaxed Newton's method given in Theorem 7 are satisfied. So, relaxed Newton's method starting form $x_{0} \in B\left(x_{0}, 1\right)$ converges to the solution of (54). 
Example 3. Local convergence. Let $X=Y=\mathbb{R}^{3}, D=U(0,1), x^{*}=$ $(0,0,0)$ and define function $F$ on $D$ by

$$
F(x, y, z)=\left(e^{x}-1, y^{2}+y, z\right) .
$$

We have that for $u=(x, y, z)$

$$
F^{\prime}(u)=\left(\begin{array}{ccc}
e^{x} & 0 & 0 \\
0 & 2 y+1 & 0 \\
0 & 0 & 1
\end{array}\right) .
$$

Using the norm of the maximum of the rows and (58)-(59) we see that since $F^{\prime}\left(x^{*}\right)=\operatorname{diag}\{1,1,1\}$, we can define parameters

$$
\begin{aligned}
& b=e, \\
& \beta=2
\end{aligned}
$$

and

$$
\rho=\min \left\{1, \frac{2(1-|1-\lambda|)}{e+(2+|1-\lambda|) 2}\right\} .
$$

Then for each $0<\lambda<2$, the relaxed Newton's method (6) starting form $x_{0} \in B\left(x^{*}, \rho\right)$. Notice that the radii of this ball is greater or equal than the one only using Lipschitz condition.

Acknowledgments. The research is supported by the grant MTM2011-28636C02-01.

\section{References}

[1] J. Appell, E. de Pascale, J. V. Lysenko, and P. P. Zabrejko, New results on NewtonKantorovich approximations with applications to nonlinear integral equations, Numer. Funct. Anal. Optim. 18 (1997), no. 1-2, 1-17.

[2] I. K. Argyros, A new convergence theorem for the inexact Newton method based on assumptions involving the second Fréchet derivative, Comput. Math. Appl. 37 (1999), no. $7,109-115$.

[3] _ A semilocal convergence analysis for directional Newton methods, Math. Comput. 80 (2011), no. 273, 327-343.

[4] I. K. Argyros and S. Hilout, On the convergence of inexact Newton-type methods using recurrent functions, Panamer. Math. J. 19 (2009), no. 1, 79-96.

[5] - Inexact Newton methods and recurrent functions, App. Math. 37 (2010), no. $1,113-126$.

[6] Weaker conditions for the convergence of Newton's method, J. Complexity 28 (2012), no. 3, 364-387.

[7] _ Estimating upper bounds on the limit pointss of majorizing sequences for Newton's method, Numer. Algor. 62 (2013), no. 1, 115-132.

[8] _ On the semilocal convergence of damped Newton's method, Appl. Math. Comput. 219 (2012), no. 5, 2808-2824.

[9] I. K. Argyros, Y. J. Cho, and S. Hilout, Numerical Method for Equations and Its Applications, CRC Press/Taylor and Francis, New York, 2012.

[10] Z.-Z. Bai and J.-L. Dong, A modified damped Newton method for linear complementarity problems, Numer. Algorithms 42 (2006), no. 3-4, 207-228. 
[11] X. J. Chen and L. Q. Li, A parameterized Newton method and a quasi-Newton method for nonsmooth equations, Comput. Optim. Appl. 3 (1994), no. 2, 157-179.

[12] R. S. Dembo, S. C. Eisenstat, and T. Steihaug, Inexact Newton methods, SIAM J. Numer. Anal. 19 (1982), no. 2, 400-408.

[13] R. Fontecilla, T. Steihaug, and R. A. Tapia, A convergence theory for a class of quasiNewton method for constrained optimization, SIAM J. Numer. Anal. 24 (1987), no. 5, 1133-1151.

[14] B. I. Epureanu and H. S. Greenside, Fractal basins of attraction associated with a damped Newton's method, SIAM Rev. 40 (1998), no. 1, 102-109.

[15] X. Guo, On semilocal convergence of inexact Newton method, J. Comput. Math. 25 (2007), no. 2, 231-242.

[16] L. V. Kantorovich and G. P. Akilov, Functional Analysis, Pergamon Press, Oxford, 1982.

[17] F. V. Haeseler and H. Kriete, Surgery for relaxed Newton's method, Complex Variables Theory Appl. 22 (1993), no. 1-2, 129-143.

[18] B. T. Polyak, Newton-Kantorovich method and its global convergence, J. Math. Sci. (N. Y.) 133 (2006), no. 4, 1513-1523.

[19] J. M. Ortega and W. C. Rheinboldt, Iterative Solution of Nonlinear Equations in Several Variables, Academic Press, New York, 1970.

[20] A. M. Ostrowski, Solution of Equations and Systems of Equations, Academic Press, New York, 1966.

[21] Solution of Equations in Euclidean and Banach Spaces, Academic Press, Nueva York, 1973.

[22] L. B. Rall, Computational Solution of Nonlinear Operator Equations, Robert E. Krieger Publishing Company, Inc., California, 1979.

[23] W. Shen and C. Li, Kantorovich-type convergence criterion for inexact Newton method, Appl. Numer. Math. 59 (2009), no. 7, 1599-1611.

[24] T. Steihaug, Quasi-Newton methods for large scale nonlinear problems, Ph.D Thesis, Res. Rep. 49, School of Organization and Management, Yale University, New Hacen, CT, 1980 .

[25] J. F. Traub, Iterative Methods for the Solution of Equations, Prentice-Hall, New Jersey, 1964.

[26] S. Weerakon and T. G. I. Fernando, A variant of Newton's method with accelerated third-order convergence, Appl. Math. Lett. 13 (2000), no. 8, 87-93.

[27] T. Yamamoto, Historical developments in convergence analysis for Newton's and Newton-like methods, J. Comput. Appl. Math. 124 (2000), no. 1-2, 1-23.

[28] T. J. Ypma, Historical development of the Newton-Raphson method, SIAM Rev. 37 (1995), no. 4, 531-551.

[29] _ Local convergence of inexact Newton methods, SIAM J. Numer. Anal. 21 (1984), no. 3, 583-590.

IOANNIS KONSTANTINOS ARgYros

Department of Mathematics Sciences

Cameron University

LAWTON, OK 73505, USA

E-mail address: iargyros@cameron.edu

José Manuel Gutiérrez

Department of Mathematics and Computation

UNIVERSITY OF LA RIOJA

C/ Luis de UlloA s/n. 26004 Logroño., Spain

E-mail address: jmguti@unirioja.es 
Ángel Alberto Magreñán

Department of Mathematics and Computation

UNIVERSITY OF LA RIOJA

C/ Luis de Ulloa S/N. 26004 Logroño., Spain

E-mail address: alberto.magrenan@gmail.com

Natalia Romero

Department of Mathematics and Computation

UNIVERSITY OF LA RIOJA

C/ Luis de Ulloa s/n. 26004 Logroño., Spain

E-mail address: natalia.romero@unirioja.es 